\title{
Dynamics of Literary Texts And Diffusion
}

\author{
Agamirza E. Bashirov \\ Department of Mathematics, Eastern Mediterranean University, \\ Gazimagusa, Mersin 10, Turkey (agamirza.bashirov@emu.edu.tr) and \\ Institute of Cybernetics, National Academy of Sciences, Baku, Azerbaijan \\ Gunesh Bashirova \\ Department of Turkish Language and Literature, \\ Eastern Mediterranean University, Gazimagusa, Mersin 10, Turkey \\ (gunesh.bashirova@emu.edu.tr) \\ and Institute of Manuscripts, National Academy of Sciences, Baku, Azerbaijan
}

\begin{abstract}
There are two major views to literature. The first and older one treats literature as something, invented by human for cultural needs. The traditional studies in literature employ this view evaluating cultural value of literature. The other and new one considers literature as a part of the cybernetic system nature-human-literature, where a human play a role of a transmitter, nature is its input and literature is output. Accordingly, it presents human as a medium, communicating fundamentals of nature in the form of literary texts. Although this view is already formed, its scientific implications are not yet investigated. In this paper we employ the second view and show that literature in the whole and diffusion behave similarly. We propose a method for creation of sample paths of the diffusion process, related to literary texts. The paper is addressed to those who are interested in mathematical modeling, philosophy of literature and communication. It has a speculative form with a minimum number of mathematical formulae.
\end{abstract}

Key words: Brownian motion, diffusion, entropy, Monmouth, literary text, mathematical modeling. 


\section{Introduction: a view to literature as a diffusion process}

To describe natural and social worlds, scientists observe and collect facts, classify them and after that construct theories, covering all or major cases, with the help of corresponding mathematical models. At present there are numerous attempts to describe the structure of literary texts, while no relevant mathematical model is suggested.

For a long time literature was treated as an invention of human for cultural purposes (Frazer, 1922). This viewpoint was a consequence of the obvious role of human in forming literature. A new viewpoint had been formed in the second half of the 20th century. Analyzing a huge number of myths (primitive literary texts), collected throughout the world, scholars observed that they are similar everywhere. This led to a very important idea that literature is not a human's invention; rather it is a reflection of nature in a narrative form, which is transmitted through human (Campbell, 1993; Meletinsky, 2000). This new vision presents literature as a part of the cybernetic system nature-human-literature, where human plays a role of a transmitter, nature is its input and literature is output. Accordingly, it presents human as a medium, communicating fundamentals of nature in the form of literary texts. This vision is significant because it opens the door to mathematical investigations in the area of literature. In this paper our aim is to present evidences, showing that literature can be treated as a specific diffusion process, and suggest a method for a construction of its sample paths.

Although human has experiences with the diffusion phenomenon since its early days (for example, smelling distant things), the first related scientific record was accounted at the beginning of the 19th century. In 1827 Scottish botanist Robert Brown observed that a microscopic particle, suspended in liquid, makes a very irregular movement. This observation was reported in (Brown, 1828).

For a long time science was not able to explain the nature of the Brown's observation. An adequate account was provided in (Einstein, 1905; Smoluchowski, 1906) in terms of 
random fluctuations of a microscopic particle under a combined bombardment by liquid molecules. Thus, a Brownian motion was related to the diffusion phenomenon.

Scientists discovered that Brownian motion is also present in other spheres which are seemingly not connected to the diffusion phenomenon. For example, in the groundbreaking work (Bachelier, 1900) on asset prices at the Paris Stock Exchange, the market fluctuations, affecting the price of an asset, are considered as a Brownian motion. Later in (Black and Scholes, 1973; Merton, 1973) the Bachelier's idea was exploited in a new application of mathematics in finance, called option pricing theory.

Another field, extensively applying the concept of Brownian motion, is space engineering. The radio signals running from the ground radars to the satellites and vice versa propagate through the charged particles of the ionosphere, the water vapor in the troposphere etc. These factors affect the radio signals and create noises, behaving as a Brownian motion. In (Kalman, 1960; Kalman and Bucy, 1961) a powerful mathematical method for filtering such noisy signals was proposed.

Various studies in the fields of biology, chemistry, geology etc. demonstrated the adequacy of the Brownian motion concept for an examination of the natural processes. Therefore, nowadays the term of diffusion process is used not only for processes, which are directly related to the diffusion phenomenon. It is also used for processes, which can be studied by means of Brownian motion.

A Brownian motion has been treated mathematically since the pioneering work (Wiener, 1923), where the Brown's observation was examined in the standard case of homogenous liquid without any drift contribution to the movement. Wiener viewed it as a collection of all possible continuous paths, assigning different weights to them in accordance to the standard case. Nowadays, this weighed collection of continuous paths is referred to as a Wiener process. We refer to (Gihman and Skorokhod, 1969; Liptser and Shiryayev, 1977; Davis, 1977; Bashirov, 2003) for a discussion of Wiener processes. 
The issue of the diffusion phenomenon was resumed in (Ito, 1944; Ito, 1951) by creating stochastic calculus that considers a diffusion process as a combination of its drift and diffusion components, the latter being driven by a Wiener process. This calculus is a mathematical tool for the option pricing, Kalman-Bucy filtering theories and their generalizations.

Can literature be related to diffusion? At first glance, this looks improbable. However, there are many intriguing parallels between them. Indeed, a literary text can be treated as a sample dynamical process.

To explain this idea, notice first that in spite of the multifarious nature of literature, improving and promoting the reader's social conscience, culture, ethical and aesthetical value, we confine our discussion only to its structure. For us, a literary text is a sequence of events, forming its plot. This means that every literary text develops by running over its events. Numbering these events sequentially from the first to the last one, we can say that there is a discrete clock within every literary text, which runs from 0 (the starting event) to $n$ (the last event). In fact, this is a clock that fixes $n$ instants of continuously running time. "The state of a literary text", the exact meaning of which will be formulated later, changes from event to event, i.e., varies as time runs. Thus, the structure of every literary text is indeed a dynamical process, a process with the state changing in time.

On the other hand, it is true that not every sequence of events makes a literary text. In a literary text events are logically connected, which implies that all literary texts share a common structure. This suggests a selection of one of the literary texts with the purpose to consider others as its deviations. It is not even necessary to have this specific literary text among really existing ones. It can be generated artificially as well. But it is very important to have it as simple as possible, free of deliberate noise peculiar to other literary texts. These deviations allow viewing literature as a random phenomenon, in which literary texts are sample cases. 
The dynamical structure and the randomness present the collection of all literary texts as a random process. Taking also into consideration the fact that the literary texts in the main are written by independent authors, we can conclude that this random process is of diffusion type, driven by a Wiener process.

The following lists some preliminary intriguing parallels between literature and diffusion:

- The cybernetic system nature-human-literature has a more obvious analog in the case of diffusion, in which human transmits chaos and order in molecular level and constructs a mathematical model of diffusion. In a simple form, this is Wiener's model of the Brownian motion, i.e., a Wiener process. More generally, this model is described by Ito's diffusion equation.

- Diffusion is modeled as a collection of paths. This idea lies on the base of Wiener's model of the Brownian motion. Similarly, literature can be regarded as a collection of literary texts.

- Every diffusion path concerns a microscopic particle. In a very similar way, every literary text has a hero.

- A microscopic particle makes motion by colliding with the molecules of liquid or gas. Analog of this in literature is the interaction of the hero and the medium described in a literary text.

- Every diffusion process has a drift component, reflecting the movement of the particle together with the medium under the action of external forces. In literature this issue is reflected by the existence of the common structure, which is peculiar to every literary text.

- Every diffusion process has a diffusion component, caused by internal collisions of the particle with the molecules of medium. Similarly, distinct literary texts deviate from each other by the distinct sequences of events.

- The paths of every diffusion process are waited in accordance to the probability of their occurrence. This is parallel to the natural classification of the literary texts to be masterpieces, tolerably written, forgettable etc. 
The weights assigned to the literary texts in accordance to the reader's objective classification define a probability distribution on the collection of all literary texts. This suggests a diffusion process, answering to this probability distribution and describing the structure of literary texts. Then its path represents a literary text.

Notice that the way of interpretation of literary texts as a random process is not new in the community of literary philosophers. For example, in (Paulson, 1988) literary texts have been treated as information in a noisy channel. Such attempts of interpretation in languages of natural sciences and mathematics enlarge the domain of literary discussions.

In this paper we use a speculative form of presentation with minimal number of mathematical formulae in order to make it accessible to natural scientists as well as to literary philosophers. Since the interdisciplinary nature of the paper, we give necessary information from related fields.

\section{Cosmology and Literature}

The drift component of the diffusion process, which we hypothetically assign as a mathematical model for literary texts, is closely related to cosmology.

One of the theories in cosmology considers the Universe as a substance emerging from a primordial highly compressed and infinitely hot point at some time in the past. This totally ordered initial state caused a strong explosion, called the Big Bang. Ever since the initial order has been particularly transforming to chaos and this process has been continuing because the universe is still expanding. At present, it is a combination of chaos and order, becoming gradually more chaotic and less ordered. The universe is still in its early stages of development. Its age since the Big Bang has been estimated to be about 15 billion years. Although this is stupendously long by human standards, it is not longer in comparison with the age of the oldest surface rocks, found in the Greenland mantle, which are estimated to be 3.9 billion years old. Therefore, scientists conclude that the universe is somewhat chaotic, mostly being ordered though. A converse process, 
transformation of chaos to order, is expected to start when the entropy of the Universe reaches its maximal value. This convergent process will continue until the Universe will again collapses into a point. Consequently, one existential period of the Universe will be completed, giving rise to the next period. This great cycle of the Universe is graphically displayed in Figure 1. We refer to (Hawking, 1990; Barrow, 1994) for this cosmological theory.

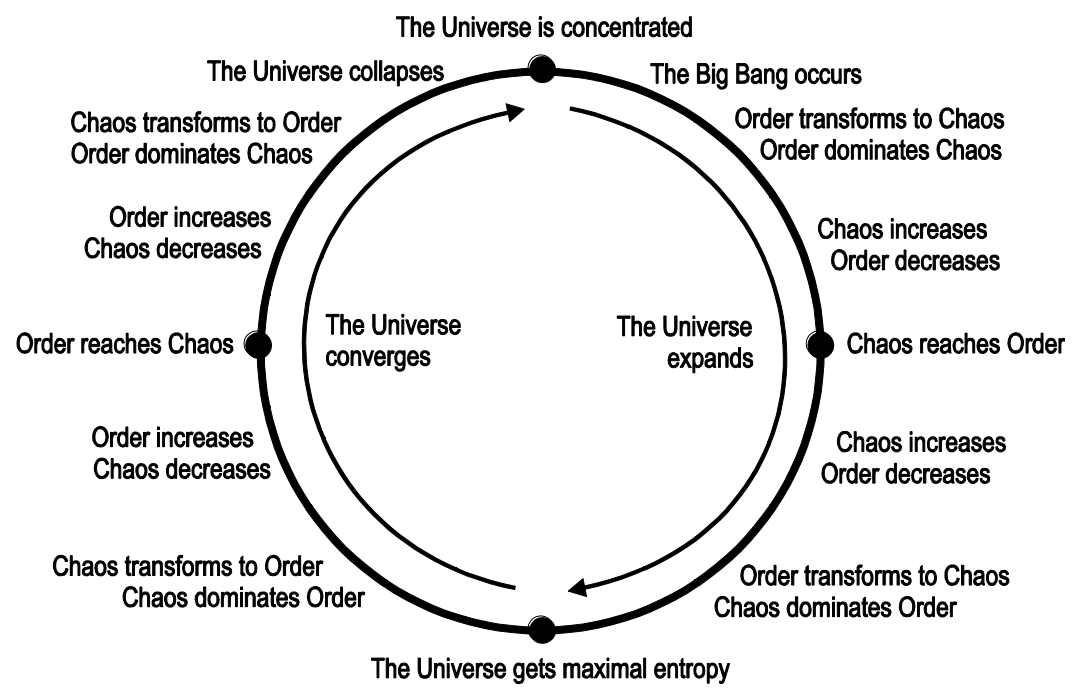

Figure1: The Great Cycle of the Universe.

Turning to literature, a parallel can be drawn with an Arabian tale on fisherman and genie. While the genie is in the brass bottle, it can not act at all and, therefore, is in the totally ordered state. As soon as the genie is freed from the bottle, its unpredictable movements increase chaos all over the place. Thus, the Arabian tale is similar to the Great Cycle of the Universe: the fisherman opens the bottle (the Big Bang occurs and the Universe comes into existence), the genie escapes the bottle (chaos in the expanding Universe increases), the fisherman tries to drive the genie back into the bottle (the Universe converges, becoming less chaotic) and the genie is back to the bottle (the Universe collapses). 
The above comparison of the Universe and one particular literary text is not accidental. The Universe is self-similar, i.e., iterates itself (its Great Cycle) in its small parts of all levels: the solar system, atoms, movements in atmosphere and in oceans, plate tectonics and rock cycle etc., all of them behave in a cyclic manner. And it should not be a surprise that a similar iteration takes place in literary texts. Indeed, the plot of a literary text describes events, which unfold at the beginning, then rapidly develop, finally come to a denouement. Based on this, some scholars, such as Meletinsky, believe that literary texts are structurally parallel to the Great Cycle of the Universe. They argue that there is one core plot for all literary texts and it is a property of the Nature. A human manifests this core plot or its parts with more or less deviations in order to direct it to specific social, ideological and cultural problems in the society. This is similar to an application of the law of gravitation (a property of the Nature) to solve technical problems such as flying aircrafts, orbiting satellites etc. This challenging issue is still being widely discussed by scholars.

We attempt to apply this theory to derive the drift component of the diffusion process, describing the structure of literary texts. For this, we will state a mathematically idealized primitive literary text, calling it a prime. It is already outlined by literary philosophers. Next, for more details, we enter to the world of myths.

\section{The Monomyth}

Myths, being present in all societies with more or less oral traditions, formally form a genre in literature. Therefore, they share the same structure with other literary texts. However, there are specific features, distinguishing myths from other literary texts. Indeed, myths are the first outcomes of thought in the lineage of human evolution, communicating fundamentals of the nature and societies in symbolic form of mystical experience of God in timeless past. Other literary texts communicate human experience in visible time. Literature (and other forms of art as well as science) inherited functions and themes of myths. We refer to (Meletinsky, 2000) for a very nice introduction to myths and relevant theories. 
The original meaning of the word "myth" (mythos in Greek) is a fiction (invented in archaic societies). The scholars of the 19th century have also treated myths in its original meaning. A new viewpoint formed in the second half of the 20th century, according to which there is a general outline, which is common for all myths. Such an outline, called the monomyth, is given by the most famous author on myths (Campbell, 1993). For historical clearance, it should be noted that Campbell borrowed the term "monomyth" from (Joyce, 1939).

Campbell's monomyth is a circular journey of the hero, which is described in the three major steps: (a) departure, (b) initiation and (c) return, each having sub-steps. These are illustrated in Figure 2. In this figure the bottom point that is labeled as the Deep of Darkness is added by the authors of the present paper to Campbell's original picture. A comparison of Figures 1 and 2 clearly demonstrates that the monomyth and the Universe behave similarly. The only distinction is that the universe behaves in continuous time, while the monomyth in discrete.

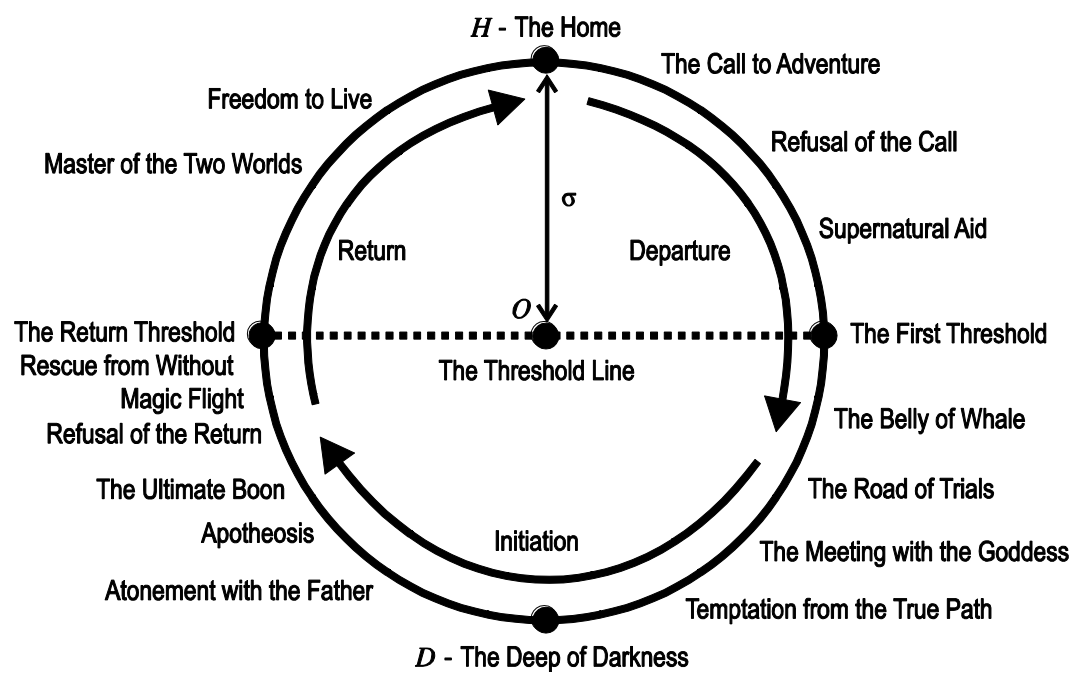

Figure 2: The Campbell's Monomyth. 
Probably, describing the monomyth as a journey along a circle, Campbell meant a finite number of discrete jumps along a closed smooth curve, which takes the hero back to the starting point - the Home. Denote this hypothetical curve by $C$. We define our prime as an ordered set of continuum number of events placed as points on the curve $C$. Note that the prime includes all points of $C$, while the monomyth consists of a finite number of them, which correspond to its events in the order of their occurrence. The points of the monomyth, joined sequentially to each other, produce a polygon inscribed to $C$. Therefore, the monomyth is indeed an approximation to the prime.

The circular shape of $C$ in Figure 2 is questionable. But what is definite is that the journey takes place around some point $O$ located below the point $H$ (the Home). This means that the curve $C$ starts and ends at $H$, passing the point $D$ (the Deep of Darkness), but it does not intersect the line segment $\mathrm{OH}$ during the journey. Let $\sigma$ be the length of $O H$. Thus the points $O$ and $D$ depend on $\sigma$, but $H$ does not.

To describe the prime mathematically we need two variables in order to establish a relation between them. One of these variables should preferably express a naturally running quantity. If the monomyth describes a sequence of $n$ events, then such a variable may be an integer running from 0 to $n$. At each event the hero's location is at some point on $C$ and intention is to turn about $O$. Therefore, we can set up the numbers $0=\theta_{0}<$ $\theta_{1}<\cdots<\theta_{n}=2 \pi$ so that $\theta_{k}$ equals to the angle between $O H$ and the line segment joining $O$ and the $k$ th location of the hero. Since the prime is defined as a continuous version of the monomyth, it is suitable to take the angle $\theta$ continuously varying from 0 to $2 \pi$, as a naturally running variable.

The other variable should quantify the state of the prime. The theory, described in Section 2 , suggests that there are two parties in every literary text. Informally, we can refer to them as the order and the chaos. A literary text can be viewed as starting with a full domination of the order over the chaos. But this domination is unstable. As the plot 
develops, little by little the order loses its power and the chaos becomes more and more powerful. There is a moment in the literary text when everything becomes most complicated, i.e., the chaos gets a full domination over the order. Then the reverse process occurs; the order little by little recovers its power and the literary text ends again with a full domination of the order over the chaos. Indeed, the role of the hero in this process reduces to the role of a float, indicating the relation between the order and the chaos at the events of the literary text. All these concern the prime as well since it is a literary text though it is primitive and idealized.

The distance from $O$ to the point on the curve $C$ corresponding to the current value of $\theta$ reflects the current relation between the order and the chaos. Shorter is this distance, closer is the hero to the point $O$. Respectively, the hero has an opportunity to finalize quickly its mission of turning about the point $O$. This contributes to the order. On the contrary, longer is this distance; far is the hero from $O$, implying that it needs a lengthy time to finalize its mission. This contributes to the power of the chaos. Therefore, the distance from $O$ to the point on the curve $C$, corresponding to the current value of $\theta$, can be taken as a variable that quantifies the state of the prime. Let this distance be $R_{\sigma}(\theta)$. Below we will see that it indeed depends on $\sigma$.

\section{The Drift Component}

In this section we heuristically deduce the function $R_{\sigma}$ and take it as a drift component of the diffusion process, describing the structure of literary texts. The first condition on $R_{\sigma}$ should be $R_{\sigma}(0)=R_{\sigma}(2 \pi)=\sigma$, which means that the journey anyway starts and ends at the point $H$.

Denote by $R_{\sigma}^{*}(\theta)$ a positive number which shows how many times $R_{\sigma}$ changes (becomes shorter or longer) at $\theta$. Unlike the derivative $R_{\sigma}^{\prime}(\theta)$, showing the rate of change of $R_{\sigma}$ at $\theta, R_{\sigma}^{*}(\theta)$ is defined as the limit 


$$
\lim _{\Delta \theta \rightarrow 0}\left(\frac{R_{\sigma}(\theta+\Delta \theta)}{R_{\sigma}(\theta)}\right)^{\frac{1}{\Delta \theta}}
$$

and called a multiplicative derivative or *derivative of $R_{\sigma}$ at $\theta$. We refer to (Bashirov et al., 2008) for a discussion of *derivatives and *integrals (generally, *alculus). Here, we only notice a simple formula

$$
R_{\sigma}(\theta)=e^{R_{\sigma}^{\prime}(\theta) / R_{\sigma}(\theta)},
$$

linking $R_{\sigma}^{*}(\theta)$ and $R_{\sigma}^{\prime}(\theta)$.

From the nature of the monomyth, in the first half of the journey, when the hero is motivated for adventures, any adventure implies more adventures. Therefore, $R_{\sigma}^{*}$ must be comparable with the positive powers of $R_{\sigma}$. but, in the second half, when the hero's intention is in the direction of going The Home, any adventure implies less adventures. Therefore, $R_{\sigma}^{*}$ becomes comparable with the positive powers of $1 / R_{\sigma}$. So, we can state the *differential equation

$$
R_{\sigma}^{*}(\theta)=R_{\sigma}(\theta)^{\lambda(\pi-\theta)}, 0 \leq \theta \leq 2 \pi,
$$

with $\lambda>0$ being a parameter, where $R_{\sigma}^{*}(\theta)$ is directly proportional to $R_{\sigma}(\theta)$ with a positive decreasing degree when $0<\theta<\pi$, it is inversely proportional to $R_{\sigma}(\theta)$ with a negative decreasing degree (i.e., directly proportional to $1 / R_{\sigma}(\theta)$ with a positive increasing degree) when $\pi<\theta<2 \pi$, and it equals to 1 at $\theta=\pi$ when the intention to adventures changes.

It is not difficult to verify that the solution of the equation in (1) is $R_{\sigma}(\theta)=e^{c e^{\lambda \theta(2 \pi-\theta) / 2}}$.

After verifying the condition $R_{\sigma}(0)=R_{\sigma}(2 \pi)=\sigma$, we specify $c=\ln \sigma$, i.e., $R_{\sigma}(\theta)=e^{(\ln \sigma) e^{\lambda \theta(2 \pi-\theta) / 2}}=\sigma^{e^{\lambda \theta(2 \pi-\theta) / 2}}, \quad 0 \leq \theta \leq 2 \pi$.

Thus, the shape of the curve $C$ is described by (2) and it depends on $\sigma$. Here, the value $\sigma$ $=1$ produces $R_{1}(\theta) \equiv 1,0 \leq \theta \leq 2 \pi$, i.e., $C$ becomes a unit circle. Figure 3 shows the curve $C$ for $\sigma=0.99, \sigma=1$ and $\sigma=1.01$ at $\lambda=1$. Note that the point $D$, indicating the 
Deep of Darkness, goes down catastrophically for even small increase of $\sigma=1$. For example, if $\sigma=1.1$, then the respective points $O$ and $D$ are apart each other for approximately 570000 units. At the same time $D$ becomes very close to $O$ for small decrease of $\sigma=1$. For example, if $\sigma=0.9$, the respective points $O$ and $D$ are almost coincide.

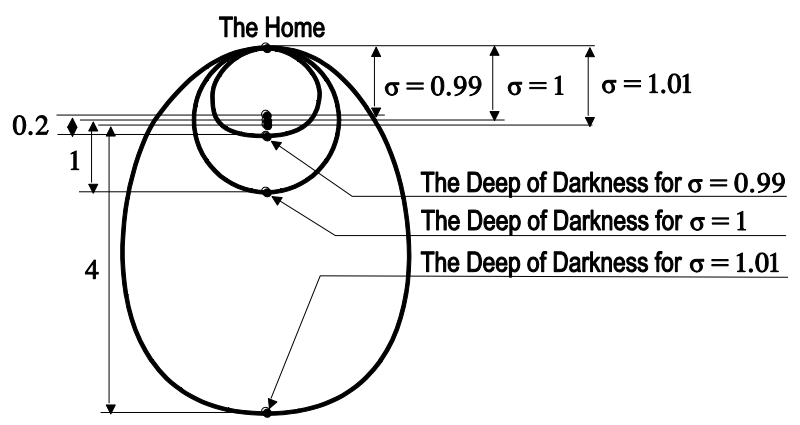

Figure 3: The curve $C$ for $\sigma=0.99, \sigma=1$ and $\sigma=1.01$ at $\lambda=1$.

This abnormality can be compensated by respective selection of $\lambda$, i.e., $\lambda=\lambda(\sigma)$ and $\sigma=$ $\sigma(\lambda)$ in which functions are decreasing; $\sigma>1$ requires $\lambda<1$ and $\sigma<1$ requires $\lambda>1$. Leaving the challenging issue on relation between $\sigma$ and $\lambda$ free for investigations, we conclude that the prime is not unique, it depends on $\sigma$ and $\lambda$, which somehow classify the literary texts. This classification seems to be parallel to the genre classification, but we prefer to call it as category rather than genre. Among these values, $\sigma=1$ is most stable; producing $R_{\sigma}(\theta) \equiv 1$ independently on $\lambda$, and most probably covers a huge part of literary texts if not all. At least, for Campbell's monomyth and for myths in general, which are moderate (golden ratio) journeys along the circle $C$, it should be taken $\sigma=1$. So, Campbell's guess about the shape of $C$ is seen to be correct. We can only add that its radius equals to 1 . The function in (2) will be taken as a drift component of the diffusion process, describing the structure of literary texts.

\section{The Diffusion Component}


What is the distinction between a sample literary text and the corresponding prime? In the prime, $R_{\sigma}$ changes smoothly since it is a literary text with a minimal stress in all samples, corresponding to a specific value of $\sigma$. But, in a sample literary text, $R_{\sigma}$ changes non-smoothly. The hero, interacting with the medium of the literary text, gets a series of small successes and failures within small units of length such as chapters, sections, subsections etc. Namely, this property makes literary texts interesting and attractive for the reader. Failures momentarily stretch out the paths of heroes apart the curve $C$ (a tendency, increasing amount of chaos and, hence, making a literary text lengthy), whereas successes compress them toward the same curve $C$ (a tendency, resolving existing chaos and, hence, shortening a literary text). Therefore, the plots of literary texts can be regarded as zigzagging closed paths. In fact, the corner points of them answer to the events of literary texts since they occur discretely, but we join the corner points to get continuous zigzagging paths.

To put all these into the equation in (2), consider the collection of all literary texts (written and those which can be written) as a sample space $\Omega$. The element $\omega \in \Omega$ is then a sample literary text, and $R_{\sigma}$ from (2) should be replaced by $r(\theta, \omega)=R_{\sigma(\omega)}(\theta, \omega)$, indicating that $r$ depends on a current $\theta$ as well as on a sample literary text $\omega$. Here, $r$ depends on $\omega$ not only through $\sigma$ (or through $\lambda$ ). The value of $r$ varies within a literary text, depending on a momentary increase or decrease of the amount of chaos and these variations at $\theta$ are not same for different literary texts. A suitable form for the random function $r$ is

$$
r(\theta, \omega)=\sigma(\omega)^{e^{\lambda(\omega) \theta(2 \pi-\theta) / 2} \eta(\theta, \omega), \quad 0 \leq \theta \leq 2 \pi, \quad \omega \in \Omega,}
$$

where the random disturbance is integrated as a multiplication by another random process $\eta$. The requirements on $\eta$ are as follows:

- At the initial and end values $\theta=0$ and $\theta=2 \pi, r$ is random only through $\sigma$ or $\lambda$. This is because at the beginning and at the end of every literary text the order has full domination over the chaos. Respectively, $\eta(0, \omega)=\eta(2 \pi, \omega)=1$. 
- The factor $R_{\sigma(\omega)}(\theta, \omega)=\sigma(\omega)^{e^{\lambda(\omega) \theta(2 \pi-\theta) / 2}}$ corresponds to the simplest literary text, i.e., to the prime of the category to which the sample $\omega$ belongs. For other samples of the same category, $r(\theta, \omega)$ cannot take smaller value. Respectively, $r(\theta, \omega) \geq R_{\sigma(\omega)}(\theta, \omega)$, implying $\eta(\theta, \omega) \geq 1$.

- In the sample $\omega$, a momentary increase of chaos at $\theta$ makes the value $r(\theta, \omega)$ large, and a momentary decrease of chaos at $\theta$ makes the value $r(\theta, \omega)$ small. The best way for integration of these changes to $\eta$ is through the entropy $H(\theta, \omega)$, which measures the uncertainty in the sample $\omega$ at the instant $\theta$. Note that from the properties of the entropy, we have $H(0, \omega)=H(2 \pi, \omega)=0$ and $H(\theta, \omega) \geq 0$. Combining these three items we deduce

$\eta(\theta, \omega)=e^{c H(\theta, \omega)}$,

where $c$ is a positive number, expressing the sensitivity of literary texts to random disturbances and functioning as the volatility in the option pricing equation (Mikosch, 1999). Thus

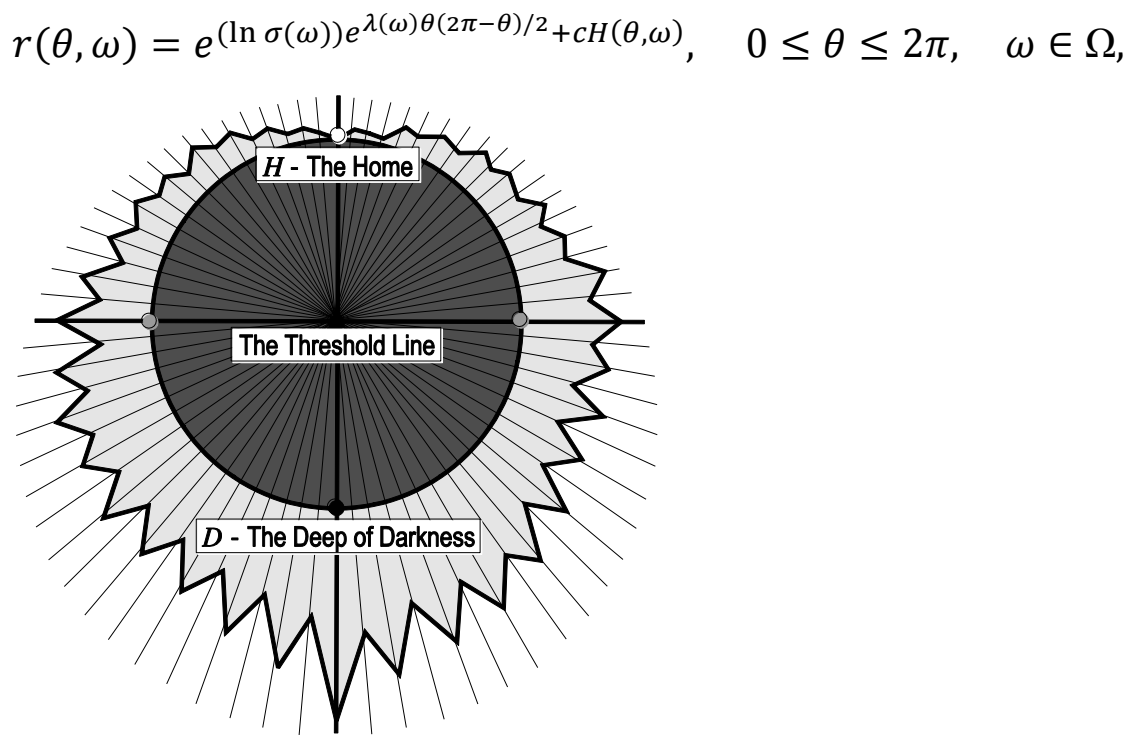

Figure 4: A zigzagging closed path associated with a literary text.

We suggest a hypothetical diffusion process, which has the sample paths as described by (3), as a mathematical model for the structure of literary texts. Figure 4 demonstrates a 
sample path of $r$ in the form of zigzagging closed path and, respectively, describes a literary text (written or not written yet). In this figure, the black region, drown as a disk, is accessible for the literary texts in the category only at its boundary points. In particular, the prime in the category runs along this boundary. The point $H$ (the Home) on the boundary is common for all literary texts. The zigzags are more sharpen about $\theta=\pi$, they are medium about $\theta=\pi / 2$ and $\theta=3 \pi / 2$, and less sharpen about $\theta=0$ and $\theta=2 \pi$.

The formula in (3) does not express the hypothetical diffusion process in terms of a Wiener process. For this, the behavior of $H(\theta, \omega)$ over a huge part of literary texts is required. But (3) allows one to create closed zigzagging paths corresponding to different literary texts. This will be demonstrated in the next section.

\section{Smooth Model}

A microscopic particle, making a Brownian motion, has a finite speed at each instant. Therefore, it can be expected that the paths of Wiener processes are differentiable. However, they are nowhere differentiable. A microscopic particle, making a Brownian motion, covers a finite distance at each time interval. Therefore, it can be anticipated that the paths of Wiener processes are rectifiable at each finite time interval. However, they have an infinite length. These and other disagreements suggest that a Wiener process (as a mathematical model) describes Brownian motion (a real process) in an ideal case when the distance between every two consequent collisions is infinitely small. This is because a typical path of Wiener process has infinitely many zigzags between its every two distinct values, though paths of a Brownian motion have large but finite number of zigzags. These disagreements were observed by engineers long ago, and so they use the concept of wide band noise along with white noise (derivative of a Wiener process in some generalized sense). In (Fleming and Rishel, 1975) it is written that the real noises are wide band and a white noise is an ideal case of wide band noises. Whenever the parameters of white and wide band noises are sufficiently close to each other, the wide 
band noise can be replaced by the white noise to make the respective mathematical model simpler.

In the light of this, the corner points of closed zigzagging paths, corresponding to events of literary texts, must indeed be joined to each other in a smooth way. This suggests the following mathematical method for studying the structure of literary texts:

- Determine the number $n$ of the events described in a sample literary text $\omega \in \Omega$. This number mainly equals to the number of length units such as chapters for novels, sections for short stories, lines or couplets for verses, paragraphs or groups of consecutive paragraphs for literary texts without explicit unit of length etc. Draw a unit circle, assuming that this literary text falls into the category $r(\omega)=1$ (otherwise, draw the graph of $R_{\sigma}(\theta)$ with $\sigma \neq 1$, selecting a suitable value of $\sigma$ as well as $\lambda$ ). Indicate the top point on the circle as the Home (see Figure 2) and place the other $n-1$ points on equal distances each from the previous one along the circle. Draw $n$ rays from the center of the circle to these $n$ points.

- Quantify small successes and failures of the hero of the sample literary text at each event. Normally, a small success should follow a small failure and vice versa, but other possibilities are not excluded as well. Quantification means a computation of the entropy $H(\theta, \omega)$ at each step $\theta$, measuring how much an event contributes to the power of the chaos or the order. A contribution to the power of the chaos determines the place of the respective point along the respective ray apart from the center of the circle, whereas a contribution to the power of the order toward it. Calculate $r(\theta, \omega)$ on the base of measured $H(\theta, \omega)$ taking $c=1$ (for example) and put a point on the ray, corresponding to current $\theta$, which has the distance $r(\theta, \omega)$ from the center of the circle.

- Create a closed zigzagging path by joining these points to each other straightforward in the order of occurrence and get a picture, similar to Figure 4. 
- Approximate the zigzagging path by closed smooth curves. Such an approximation is additionally open for investigation of the component smooth functions producing best approximation.

- It is not difficult to guess that sinusoids will be dominant as basic component functions. Therefore, it is challenging to study their spectrum or a relationship of literary texts to Fourier series. An approach similar to the use of sinusoids in the theory of music can be developed for literary texts as well. But unlike the theory of music, where the sound frequencies can be measured by proper electronic devices, a similar study for literary texts will heavily depend on an influence of subjective factors.

- Another approach can base on the geometric shape of smooth approximation which is uniquely described in terms of curvature function. Both these approaches can seemingly delineate genres, tendencies etc. in literature. Getting more and more samples should allow one to estimate the parameters $\sigma, \lambda$ and $c$, involved in (3), for different genres, tendencies etc. in literature and as well as for the structure of literature in general.

The second item, mentioned above, needs a demonstration how to compute the entropy as a measure of uncertainty at each step. As an example consider a simplified version of the above mentioned Arabian tale on fisherman and genie. This tale (denote it as a sample $\omega_{0} \in \Omega$ ) can be viewed as a sequence of the following evens:

- Event 0 - The Home. (Introduction to the tale, $\theta_{0}=0$ ) There was an old fisherman, who every day goes seaside for fishing.

- Event 1. (The adventures start, $\theta_{1}=\pi / 4$ ) One day he catches an old brass bottle in his net, opens it, and a genie has been freed angrily declaring that he will kill his rescuer.

- Event 2. $\left(1^{\text {st }}\right.$ trial, $\left.\theta_{2}=\pi / 2\right)$ In order to save his life the fisherman tells a story of a man who kills the doctor healed him of a disease.

- Event 3. $\left(1^{\text {st }}\right.$ trial is unsuccessful, $\left.\theta_{3}=3 \pi / 4\right)$ The genie does not change his intension. 
- Event 4. ( $2^{\text {nd }}$ trial, $\left.\theta_{4}=\pi\right)$ The fisherman tells another story about a prince who kills his faithful dog.

- Event 5. $\left(2^{\text {nd }}\right.$ trial is unsuccessful, $\left.\theta_{5}=5 \pi / 4\right)$ The genie is still angry, rejecting the fisherman's arguments.

- Event 6. $\left(3^{\text {rd }}\right.$ trial, $\left.\theta_{6}=3 \pi / 2\right)$ The clever fisherman uses a trick, telling that if the genie can make himself so tiny that to be placed into a small bottle.

- Event 7. ( $3^{\text {rd }}$ trial brings success, $\left.\theta_{7}=7 \pi / 4\right)$ The foolish genie demonstrates it by entering back into the bottle.

- Event 8. (The fisherman saves his life, $\theta_{8}=2 \pi$ ) The fisherman shuts up the bottle and casts it into the sea.

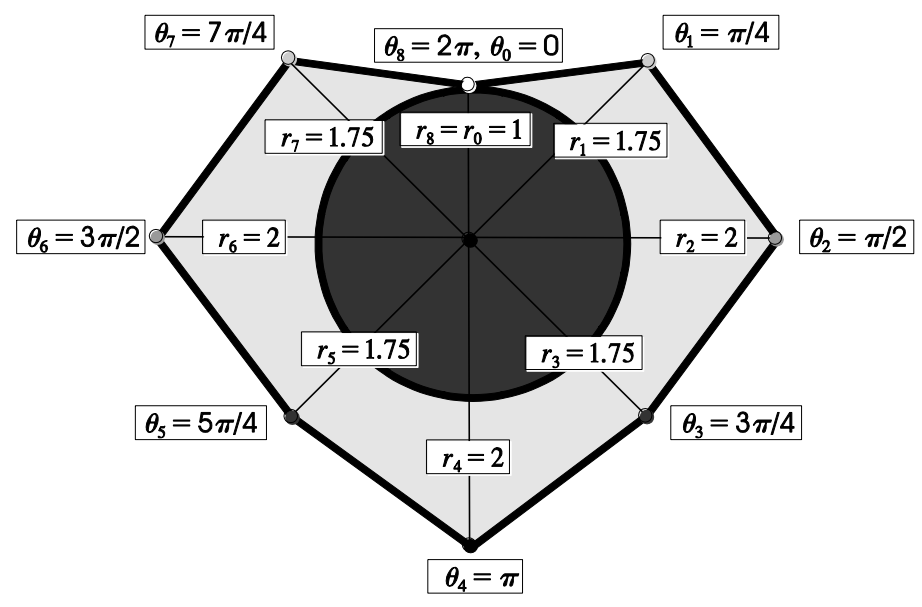

Figure 5: The Arabian tale on fisherman and genie.

In order to calculate the entropy, we assign different probabilities to two possibilities: $S$ (success, the fisherman's life is saved) and $F$ (failure, the fisherman's life is in danger). The probabilities $p=\mathbf{P}(S)$ and $q=\mathbf{P}(F)$ at every step will be arranged to be 0 (if the possibility is impossible), $1 / 4$ (if there is a small probability to occur), $1 / 2$ (if the possibility is fifty-fifty), 3/4 (if there is a great probability to occur) and 1 (if the possibility is sure). The entropy will be calculated according to the well-known formula

$$
H\left(\theta, \omega_{0}\right)=-p \ln p-q \ln q
$$

under the convention $0 \cdot \ln 0=0$. Then, assuming that $\sigma\left(\omega_{0}\right)=c=1$, we have 


$$
r\left(\theta, \omega_{0}\right)=e^{H\left(\theta, \omega_{0}\right)}=\frac{1}{p^{p \cdot q^{q}}}
$$

under the convention $0^{0}=1$. Thus,

- Step 0. $p=1, q=0 \Rightarrow r_{0}=r\left(\theta_{0}, \omega_{0}\right)=1$.

- Step 1. $p=1 / 4, q=3 / 4 \Rightarrow r_{1}=r\left(\theta_{1}, \omega_{0}\right)=4 / \sqrt[4]{27} \approx 1.75$.

- Step 2. $p=1 / 2, q=1 / 2 \Rightarrow r_{2}=r\left(\theta_{2}, \omega_{0}\right)=2$.

- Step 3. $p=1 / 4, q=3 / 4 \Rightarrow r_{3}=r\left(\theta_{3}, \omega_{0}\right)=4 / \sqrt[4]{27} \approx 1.75$.

- Step 4. $p=1 / 2, q=1 / 2 \Rightarrow r_{4}=r\left(\theta_{4}, \omega_{0}\right)=2$.

- Step 5. $p=1 / 4, q=3 / 4 \Rightarrow r_{5}=r\left(\theta_{5}, \omega_{0}\right)=4 / \sqrt[4]{27} \approx 1.75$.

- Step 6. $p=1 / 2, q=1 / 2 \Rightarrow r_{6}=r\left(\theta_{6}, \omega_{0}\right)=2$.

- Step 7. $p=3 / 4, q=1 / 4 \Rightarrow r_{7}=r\left(\theta_{7}, \omega_{0}\right)=4 / \sqrt[4]{27} \approx 1.75$.

- Step 8. $p=1, q=0 \Rightarrow r_{8}=r\left(\theta_{8}, \omega_{0}\right)=1$.

The heart-like zigzagging closed path, corresponding to the Arabian tale on fisherman and genie, is drawn in Figure 5.

\section{Concluding Remarks}

A natural question is whether two distinct literary texts can be associated with the same zigzagging path. The answer is yes if zigzags are formed on the base of events as described above. Regarding this issue, note that every event is a sequence of sentences which create small zigzags within a zigzag. Going deeper, every sentence is a sequence of words which creates tiny zigzags within a small zigzag. If zigzagging paths reflect all levels of zigzags, then definitely every two distinct literary texts have distinct associated zigzagging paths. The zigzags of the sentence and word levels reflect the technique of writing and the play of words, peculiar to the specific writer. It seems it should be difficult to record zigzags of these levels. But the zigzags of the event level are quite recordable and tell us about the macro divisions in literature such as genres, tendencies etc. The authors of the paper expect that integration of all levels of zigzags should produce fractal-type closed curves that display self-similarity on all level scales, for 
highly ranked literary texts. This can be a test for ranking of literary texts and a guide for writing highly ranked literary texts.

The following philosophical conclusion is also remarkable as well. The above theory presents the human brain as a noisy channel, inputs of which are objective observations on nature and society. But its outputs are literary texts, communications about laws of nature and society corrupted by a noise, in which connection masterpieces of literary texts correspond to laws being made substantially noisy. Accordingly, the concept of noise has a dual nature. On one hand, sciences extract the laws of nature and society from noisy observations, fighting against the parasitic behavior of noises. On the other hand, the readership ranks high the literati's outputs if they are substantially noisy, i.e., noises beautify literary texts, demonstrating beautiful behavior. This seemingly extends to arts in the whole. Respectively, two major domains of human activity, sciences and arts, become distinguished by the dual nature of noises.

Another interesting conclusion can be noticed as well. The cybernetic system naturehuman-literature, mentioned and discussed above, tells that literature contains some partial information about behavior of the universe. Hence, a mathematical study of this communication may turn to a challenging method of study in cosmology as well. For example, nowadays cosmologists are discussing whether the Big Bang was a strong explosion or a merely expansion of the Universe. The above theory supports the second one since the literary texts, especially; the myths start to develop slowly.

\section{Acknowledgement}

The paper is partially supported by B-type project MEKB-09-05. The authors also thank Prof. Sergey Khrushchev for useful discussions and suggestions.

\section{References}


Bachelier, L. (1900) Theorie de la Speculation. Annales de l'Ecole Normale Superieure $17,21-86$.

Barrow, J.D. (1994) The Origin of the Universe. Basic Books, New York.

Bashirov, A.E. (2003) Partially Observable Linear Systems under Dependent Noises. Birkhauser, Basel.

Bashirov, A.E., Kurpınar E.M., Özyapıcı A. (2008) Multiplicative calculus and its applications, J. Mathematical Analysis and Applications, 337, 36-48.

Black, F., Scholes, M. (1973) The pricing of options and corporate liabilities. J. Political Economy 81, 635-654.

Brown, R. (1828) A brief account of microscopical observations made in the months of June, July and August, 1827, on the particles contained in the pollen of plants; and on the general existence of active molecules in organic and inorganic bodies. Philos. Mag. Ann. of Philos. New Series 4, 161-178.

Campbell, J. (1993) The Hero with a Thousand Faces. Fontana Press, London.

Davis, M.H.A. (1977) Linear Estimation and Stochastic Control. Chapman and Hall, London.

Einstein, A. (1905) Die von der molekularkinetischen theorie der wärme geforderte bewegung von in ruhenden flüssigkeiten suspendierten teilchen. Ann. Phys. (Leipzig) 17, 549-560.

Fleming, W.H., Rishel, R.W. (1975) Deterministic and Stochastic Control. Springer, New York.

Frazer, J. (1922) The Golden Bough. Macmillan, New York.

Gihman, I.I., Skorohod, A.V. (1969) Introduction to the Theory of Random Processes. Saunders, New York.

Hawking, S. (1990) A Brief History of Time. Bantam Books, New York.

Ito, K. (1944) Stochastic integral. Proc. Imperial Acad. Tokyo 20, 519-524.

Ito, K. (1951) On stochastic differential equations. Mem. Amer. Math. Soc. 4, 645--668.

Joyce, J. (1939) Finnegans Wake. Viking Press, New York.

Kalman, R.E. (1960) A new approach to linear filtering and prediction theory. Trans. ASME, Ser. D (J. Basic Engineering) 82, 35-45. 
Kalman, R.E., Bucy, R.S. (1961). New results in linear filtering and prediction theory Trans. ASME, Ser. D (J. Basic Engineering) 83, 95-108.

Liptser R.S., Shiryayev, A.N. (1977) Statistics of Random Processes, 1: General Theory. Springer, New York.

Meletinsky, E. (2000) The Poetics of Myth. Garland, New York.

Merton, R.C. (1973) Theory of option pricing. Bell. J. Econ. Manag. Sci. 4, 141-183.

Mikosch, T. (1999) Elementary Stochastic Calculus with Finance in View. World Scientific, Singapore.

Paulson W.R. (1988) The Noise of Culture: Literary Texts in a World of Information. Cornell University Press, Ithaca.

Smoluchowski, M. (1906) Zur kinetischen theorie der Brownschen molekularbewegung und der suspersionen. Ann. Phys. (Leipzig) 21, 756-780.

Wiener, N. (1923) Differential space. J. Math. and Phys. 2, 131-174. 